\title{
A New Insight into the Observations and Analysis of Type Ia Supernovae
}

\author{
Qiuhe Peng ${ }^{1}$, Jingjing Liu ${ }^{2}$ \\ ${ }^{1}$ Department of Astronomy, Nanjing University, Nanjing, China \\ ${ }^{2}$ College of Science, Hainan Tropical Ocean University, Sanya, China \\ Email: syjjliu68@hntou.edu.cn
}

How to cite this paper: Peng, Q.H. and Liu, J.J. (2021) A New Insight into the Observations and Analysis of Type Ia Supernovae. Journal of Applied Mathematics and Physics, 9, 1808-1820. https://doi.org/10.4236/jamp.2021.97115

Received: April 21, 2021

Accepted: July 27, 2021

Published: July 30, 2021

Copyright $\odot 2021$ by author(s) and Scientific Research Publishing Inc. This work is licensed under the Creative Commons Attribution International License (CC BY 4.0).

http://creativecommons.org/licenses/by/4.0/

(c) (i) Open Access

\begin{abstract}
In this paper, we have given some analysis from observations of type Ia supernovae (SNIa). We find that the average total observational error of SNIa is obviously greater than $0.55^{\mathrm{m}}$. On the other hand, a popular view of circumstantial evidence for the accelerating universe comes from the comparison of theoretical models simulating the accelerating expansion of the universe with observations from the Wilkinson Microwave Anisotropy Probe (WMAP) satellite deviating from the observed isotropic temperature of the Cosmic Microwave Background (CMB). Due to the fact that the velocity space is not isotropic, then the theoretical simulations are incredibly consistent with observations from the WMAP and Planck satellites. We conclude that the anisotropy of the velocity space will inevitably lead to an anisotropic distribution of the CMB temperature, and the above indirect evidence of the cosmic acceleration is inadequate and inappropriate.
\end{abstract}

\section{Keywords}

Supernovae, Cosmology, Cosmic Microwave Background

\section{The Origin of the Cosmic Dark Energy Problem}

Accelerating expansion of the universe may be caused by dark energy, which is one of the most important ideas of researches in astrophysics and cosmology today. Accelerated by dark energy, this motion is different from the outward expansion of the universe [1]. The so-called Advanced Philips Relation (hereafter APR) was derived on the absolute magnitude statistics at maximum luminance of Type Ia supernovae (SNIa) related with both the width of light-curve ( $\Delta m 15$, the decline in magnitudes 15 days after the peak luminosity) and the variation of the color index ( $B$ - $V)$ of SNIa (e.g., [2] [3] [4] [5] [6]). The Direct evidence of 
cosmic accelerated expansion comes from observations of SNIa using the Advanced Philips Relation ([6] [7]). The distance modulus $\mu$ is

$$
\mu=5 \log (D)-5=m-M-A+k+\cdots
$$

where $D$ is the distance in the unit of pc, $m$ and $M$ are the apparent magnitude and absolute magnitude of SNIa, respectively. $A, K$, and “..." are the intergalactic extinction, the $K$-correction, and the errors caused by gravitational lensing and the peculiar motion of the host galaxy, respectively.

The APR is by far the most accurate method. However, in the processes of the explosive nucleosynthesis, we hold the opinion that $\Delta m 15$ is closely related to the quantity of radioactive nuclide produced. On the other hand, during the processes of the explosion, the color index varies with the expansion and cooling down rates of outer aerosphere. Based on the APR, SALT2 (Spectral Adaptive Light-curve Template) [5] is an SNIa light curve fitting software package. According to the data from 685 SNIa, which is from the SALT2, UNION2 [6], the system error of the absolute magnitudes of SNIa was found by minimizing $\chi^{2}$, which is the normalized quadratic sum of distance modulus residual [6]. As a result, they came to a conclusion that the expansion of the universe that began with the Big Bang is speeding up. Then, Saul Perlmutter, Brian Schmidt and Adam Reiss share the 2011 Nobel Prize in Physics for their SNIa observations indicating that the expansion of our universe is accelerating.

As well known, the most direct evidence has been provided for the presence of dark energy from SNIa cosmology. Recently, the evidence for downsizing of early-type host galaxies of the SNIa has been shown by Kang et al. (2016) [8]. Their results shown that the stellar population age is mainly responsible for the relation between host mass and Hubble residual. In the systematic uncertainties of SNIa cosmology, their results shown that the luminosity evolution is very important and plays a key role. The evidences for luminosity evolution in SNIa cosmology have also been discussed by Kang et al. (2020) [9]. Between the stellar population age at a $99.5 \%$ confidence level and SNIa luminosity after the standardization, they found a significant correlation. For the population age effect, which would inevitably cause a serious systematic bias with look-back time, the light-curve fitters used by the SNe Ia community may be not capable of correcting. They suggested that we must consider the systematic bias in SN cosmology for studying the dark energy [9]. In this paper, we have discussed the same error analysis of SNIa. One sees that the average of total observational error of SNIa is greater than $0.55^{\mathrm{m}}$. These results show that the universe may be not accelerating expansion.

\section{The Standard Candles of SNIa Becoming Invalidated}

Advances on the SNIa remnants have shown that the idea of a single accreting white dwarf model (known as the "standard model") for the SNIa explosion, which has been negated due to the researches on the Tycho's SNR from 2008 to 2010 [10] [11]. Because of the different origins of SNIa, their peak luminosities 
are no longer taken as standardized candles. Besides, it has been discovered that some remnants of SNIa are obviously asymmetric in spatial direction [12]. At the same time, some explosion simulations of SNIa showed that there may be significant asymmetry in the explosion of the SNIa [13] (to see Figure 1 in this paper). For these objects, Maeda et al. (2010) discussed the observations of SNIa and implicated a lopsided explosion mechanism. Their simulation shows that a white dwarf is initially ignited slightly away from the center (see Figure 1(a)), and a thermonuclear flame begins to consume the star (see Figure 1(b)). The burned material is hot and buoyant, and so the flame and ash quickly float upward (see Figure 1(c)), resulting in one side of the star being more completely incinerated and more rapidly expelled than the other (see Figure $1(\mathrm{~d})$ ).

Wang et al. (2013) [14] showed the evidence for two distinct populations of SNIa. More importantly, many physical problems, such as the mechanism and process of SNIa explosion, the explosive nuclear burning and the production of elements (especially radionuclides) in the thermonuclear explosion, as well as the expansion and cooling of the outer atmosphere at the time of the explosion remain undetermined.

According to the Advanced Philips Relation in Refs. [2] [3] [4] [5] [6], the research on SNIa is still in the exploratory stage, then these absolute magnitudes based on fitting the SALT2 SNIa light-curve and spectrum are the peak luminosity
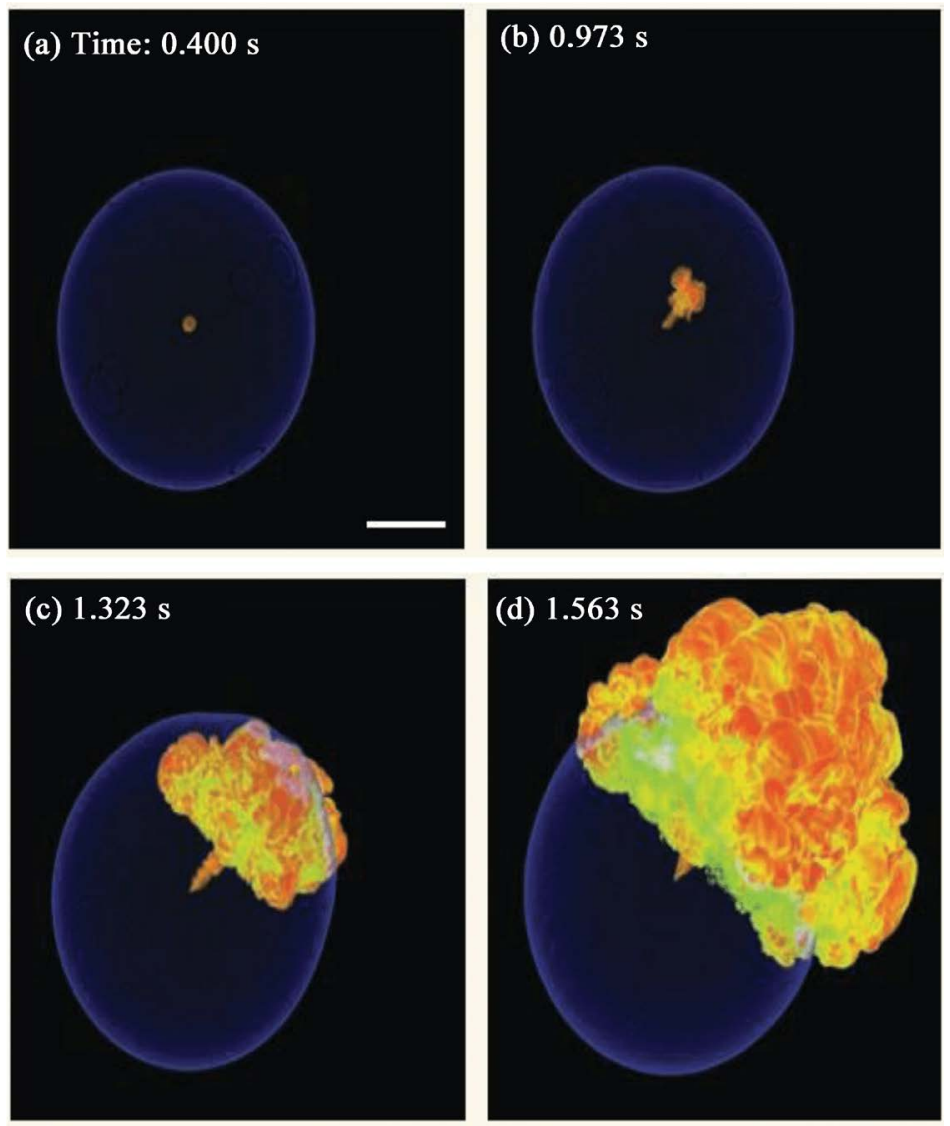

Figure 1. Lopsided supernova explosion [12] [13]. 
of 'modeling SNIa' rather than ones of real SNIa. In this paper, we will analyze the data, which is the latest and most complete SNIa compilation from UNION2.

\section{Some Serious Faults in Error Analysis of Type Ia Supernova Research Papers}

\subsection{Error in the Absolute Magnitude}

At the maximum light of SNIa, the errors of the absolute bolometric magnitude are given as follows:

1) At the maximum luminance, the absolute magnitude intrinsic error $\left(\Delta M_{\text {int }}\right)$, is just the half width at the statistic distribution curve of the number of SNIa with the maximum luminance, rather than the systematic error established using the $\chi^{2}$ check way in some papers (e.g., [1] [2] [3] [4] [5])

2) In addition to $\Delta M_{\text {int, }}$ part of $M$ comes from the transfer error caused by statistical errors of the parameters $a$ and $b$ in original Phillips relation (or of the parameters $\alpha_{x}, \beta$ in the APR), we call it as the transfer error, $\Delta M_{\text {int }}(a, b)$.

3) Part of $M$ is caused by $\Delta M_{\mathrm{obs}}$, the error of some observational quantities of both the light-curve and color index in the Advanced Phillips's way.

The total error of absolute bolometric magnitude at the maximum light $\left(\Delta M_{\text {total }}\right)$ is given by

$$
\begin{gathered}
\left(\Delta M_{\text {total }}\right)^{2}=\left(\Delta M_{\text {int }}\right)^{2}+\left(\Delta M_{\text {max }}^{\text {phillips }}\right)^{2} \\
\left(\Delta M_{\text {max }}^{\text {phillips }}\right)^{2}=(\Delta M(a, b))^{2}+\left(\Delta M_{\text {obs }}\right)^{2}
\end{gathered}
$$

where SALT2 [5] and UNION2 [6] did not give the aforementioned errors separately. They merged them into the system error caused by $\chi^{2}$-minimization. The minimum of $\Delta M_{\max }^{\text {phillips }}$ is larger than observational apparent magnitude error, $\left|\Delta M_{\max }^{\text {phillips }}\right|>|\delta m|$ (the observational error of apparent magnitude). Because the high red-shift SNIa is faint, so its $|\delta m|$ is much larger than that of nearby SNIa.

\subsection{Incorrect Systematic Error Established by Using the $\chi^{2}$ Check Test}

The error of distance modulus for the set of SNIa obeys the Gaussian distribution. It is the premise of the $\chi^{2}$ check test in this paper. However, the set of SNIa modeling doesn't really satisfy Gaussian distribution in Ref. [6]. Although the UNION2 contains $685 \mathrm{SNIa}$, the average error is $0.16^{\mathrm{m}}$, which over $10 \%$ of total SNIa are outline of $10 \sigma$. If we take a subsample including 217 SNIa with very small observational average error to do the same statistics, One finds that over $10 \%$ of total SNIa are outline of $5 \sigma$. So UNION2 shows a strong non-Gaussian distribution. Really, the critical permitted outline value of $2.6 \sigma$ in the standardized normal distribution is $0.805 \%$. Following the SATL2, performing the $\chi^{2}$ check test in the UNION2 work, the average of the total observational error of SNIa's distance modulus is $0.31^{\mathrm{m}}$. The result comes out that the $3.796 \%$ of the data is derived from $2.6 \sigma$ element with an average error of $0.31^{\mathrm{m}}$. Obviously, the 
distance modulus error deviates from the Gaussian distribution, and it is incorrect to obtain the systematic error $\sigma_{\text {sys }}$ of SNIa by the $\chi^{2}$ check test method.

We think that the real intrinsic error of an SNIa compilation should be based on statistical diagram of the number of SNIa (to see Figure 2). As we don't know the exact luminosities (the absolute bolometric magnitude) of SNIa with high redshift, it is the only way to use SALT2 to get the absolute bolometric magnitudes of "modeling SNIa". At the maximum luminosity, the intrinsic error of the absolute magnitude is the half width at half maximum of the statistic distribution curve of the number of SNIa with the maximum luminosity (to see Figure 2). The intrinsic error $\Delta M_{\text {int }}=0.38^{\mathrm{m}}$, which is larger than the systematic error given by the $\chi^{2}$ check test.

\section{Query on Accelerating Expansion of the Universe (I)}

Figure 3 shows the average total observation error of distance modulus from modeling SNIa samples as a function of red-shift, $z$. The average total observation error of distance modulus is $(\Delta \mu)_{2}=\left(\Delta M_{\text {total }}\right)_{2}+(\delta m)_{2}$. Based on the observational apparent magnitude error from the UNION2 and SNIa data, we divided intervals per $\Delta z=0.1$. By using the same $\chi^{2}$ check test method, we give the statistics to calculate this sample's average total observation error of distance modulus. One can find that the observation error of the distance modulus increases greatly with the increase of red-shift of SNIa in the very high red-shift region. As can be seen from Figure 4, the residual error of SNIa's distance modulus is a function of red-shift of SNIa. The curves of three from top to bottom represent the models of cosmic accelerating, constant speed, and slow down expansion, respectively. It is also found that the average total observational error of SNIa is obviously greater than $0.55^{\mathrm{m}}$, which is much larger than $0.40^{\mathrm{m}}$ (see in Figure 3 and Figure 4). So we don't know for sure if the universe is accelerating. Recently, Ref. [15] discussed the marginal evidence in detail for cosmic acceleration

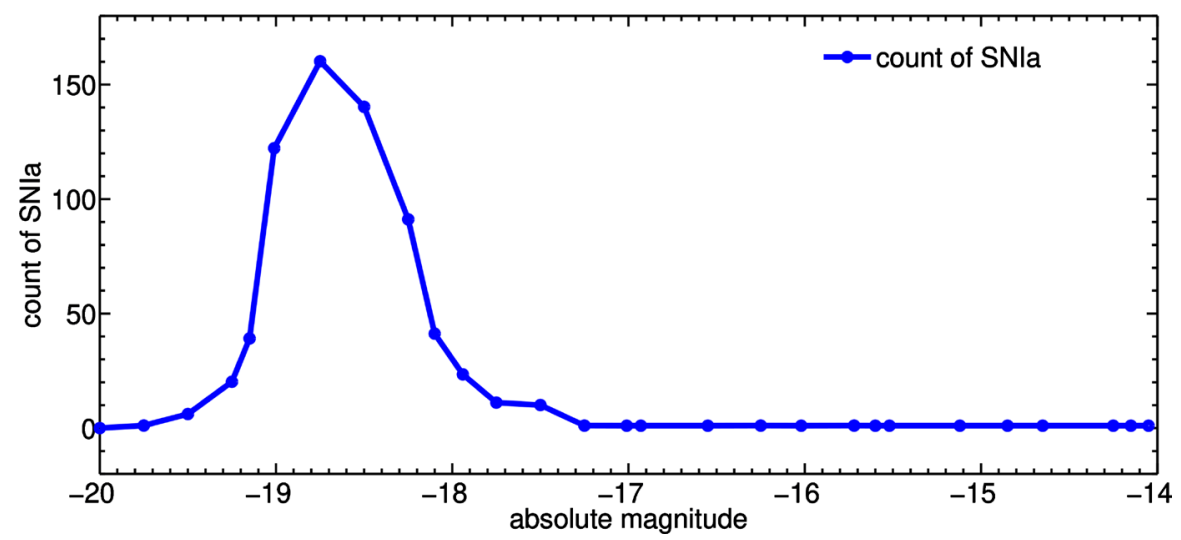

Figure 2. The Statistic distribution of the absolute magnitude at maximum luminosity $\left(M_{\max }\right)$ for SNIa. The abscissa is $M_{\max } \mathrm{f}$ SNIa, the ordinate is the number of SNIa with $M_{\max }$. The half width at the half height for the peak of the distribution curve (HMHW) is just the intrinsic error (or the proper error) of the absolute magnitude at the maximum luminosity for the samples of SNIa. 


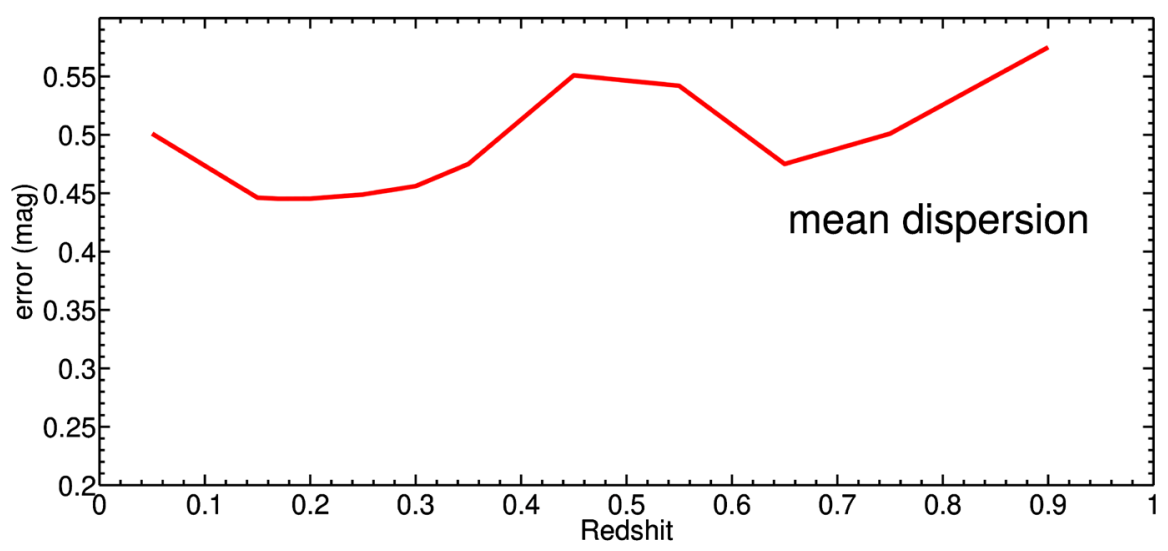

Figure 3. Varies of (average) the observational error of the distance modulus with redshift of SNIa. The abscissa is the red-shift of SNIa, the ordinate is the "modeling SNIa" sample's average total observational errors of distance modulus. It shows that the observational error of the distance modulus increases with the increasing redshift of SNIa in region of the remote high red-shift.

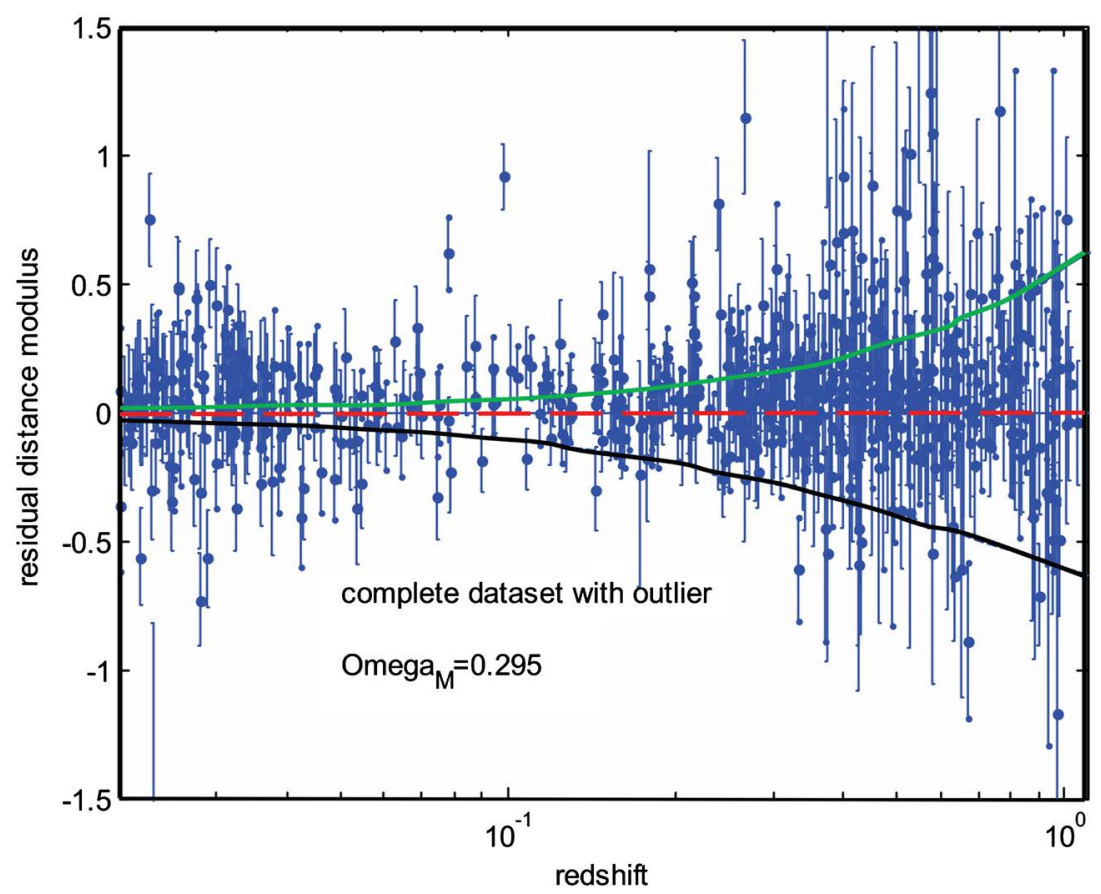

Figure 4. The residual error for the distance modulus of SNIa. The abscissa is the red-shift of SNIa, the ordinate the residual error for the distance modulus of SNIa. The three curves from left to right, from top to bottom, represent calculating curves of the universe expansion, which is accelerating, uniform or decelerating, respectively. They show that the residual error bars of the distance modulus of SNIa are too large $\left(>0.55^{\mathrm{m}}\right)$ to judge the universe expansion being accelerating, uniform or decelerating.

from type Ia supernovae. From the larger database of supernovae, they performed rigorous statistical tests to check whether these 'standard candles' really do indicate an accelerating expansion of the universe. Taking into account of the empirical procedure, by which corrections are made to their absolute magnitudes to allow for the varying shape of the light curve and extinction of dust, 
they found that the data are still quite consistent with a constant expansion rate. On the other hand, direct observational evidence of the idea of dark energy is also lost in the observational error analysis of SNIa.

\section{Anisotropy of the Observed Cosmic Microwave Background Temperature}

The prevailing idea of indirect observational evidence for the accelerated cosmic expansion is to compare theoretical simulations of the accelerated cosmic expansion with the observational data of the WMAP satellite deviating from isotropy of the observed CMB temperature. Of course, a wealth of observational data of temperature deviations from the $\mathrm{CMB}$ isotropy have been obtained by the WMAP (2003-2008) and Planck satellites (2012 up to date).

The maximum observed temperature increment deviating isotropic from the CMB temperature is $(\Delta T)_{\max }=3.346 \pm 0.0017 \mathrm{mK}$ in the direction $l=2636.85^{\circ} \pm 0.1^{\circ}, \quad b=48.25^{\circ} \pm 0.04^{\circ} \quad$ [16] (the direction of the Virgo cluster: $l=284^{\circ}$ and $\left.b=74^{\circ}\right)$. This departure, mainly caused by the Earth's motion with the velocity of $\beta=370 \mathrm{~km} / \mathrm{s} / c=0.0013$ (i.e., the dipole anisotropy) is given as

$$
\Delta T / T=T^{\prime}-T / T=-\beta^{2} / 6-\beta P_{1}(\cos (\theta))+2 \beta^{2} P_{2}(\cos (\theta)) / 3+\cdots
$$

where $P_{n}(x)$ is the Legendre polynomials, $\theta$ is the direction angle of the field point alone with the direction of the Earth's motion.

The book of Ref. [16], Weinberg described in detail the contributions to the anisotropy of the observed CMB. According to Ref. [16], we list the main contributions to the anisotropy of $\mathrm{CMB}$ as follows:

1) Departure from isotropy of the observed $\mathrm{CMB}$, arising from the Earth's motion.

2) Anisotropy due to the scattering of photons by intergalactic electrons in clusters of galaxies. (The Sunyaev-Zel'dovich effect via solution of the Kompaneets equation)

3) The primary anisotropy left over from the early universe. Afterwards, Weinberg analyzed and discussed four factors causing the primary anisotropy of the CMB. Taking out the influence of the Earth's motion, the residual anisotropy of the $\mathrm{CMB}$ temperature is about several $10^{-5} \mathrm{~K}$.

The primary anisotropy in the $\mathrm{CMB}$ temperature arises from several sources. In the eletron-nucleon-photon plasma at the time of last scattering, the corresponding red-shift, $z \approx 1.090$, the first source is the intrinsic temperature fluctuations. The second source is the Doppler effect due to velocity fluctuations in the plasma at the last scattering. The third source is the gravitational red-shift or blue-shift due to fluctuations in the gravitational potential at the last scattering. This is known as the Sachs-Wolfe effect. The last source may be the integrated Sachs-Wolfe effect due to time dependent fluctuations in the gravitational potential between the time of the last scattering and the present time.

The WMAP and Planck satellites had provided very rich observational data. 
These observational data were fitted theoretically by including all the given physical factories in some accelerated cosmic expansion models and using more than two thousands spherical harmonic functions $(I>2000)$. It seems to be very beautiful for supporting the accelerated cosmic expansion. However, it is still not complete, because an important physical factor that is anisotropic velocity space has not been taken into account to date. The anisotropy of velocity space has been shown in the velocity dispersion of galaxies for cluster of galaxies.

\section{Query on Accelerating Expansion of the Universe (II)}

\subsection{The Standard Cosmology with an Expansion of the Universe}

It is well known that the Robertson Walker metric in the standard cosmology was derived from the Einstein field equation of the general relativity based on the Mach principle with an assumption of homogeneity and isotropy everywhere. For the details, see the book of 'Gravitation and Cosmology', which was written by Ref. [17]. The Robertson Walker metric measures the scale of the expanding universe, and is given by

$$
\mathrm{d} s^{2}=-\mathrm{d} t^{2}+\alpha^{2}(t)\left(\mathrm{d} r^{2} /\left(1-k r^{2}\right)+r^{2} \mathrm{~d} \theta^{2}+r^{2} \sin ^{2} \theta \mathrm{d} \varphi^{2}\right)
$$

where the scaling factor $\alpha(t)$. According to the Friedmann equation, the CMB temperature, $T(t)$, decreases inversely with the expansion rate of the expanding universe in the standard cosmology. Thus, we have

$$
\alpha(t) T(t)=\text { const }
$$

When the velocity space is not isotropic, we may take a small anisotropy (small difference) in the velocity velocity space as a perturbation of the velocity space. A perturbation of the scaling factor will be caused by the velocity space perturbation through the energy momentum tensor in the Einstein field equation. A perturbation of the CMB temperature will be also caused by the perturbation of the scaling factor through the relation given by Equation (6).

\subsection{Anisotropy of the Velocity Space of the Universe}

$\langle|V|\rangle \approx 300-500 \mathrm{~km} / \mathrm{s}$, It is well known that the velocity dispersions of stars in the solar neighborhood in our Galaxy are given as $\sigma_{r} \approx 30-50 \mathrm{~km} / \mathrm{s}$, $\sigma_{\varphi} \approx 2 / 3 \sigma_{r}, \sigma_{z} \approx \sigma_{r} / 2$. They are anisotropic and the scope of the anisotropy is about $(30-50) \%$. All the galaxies including our Galaxy have angular momentums, which are anisotropic.

Similarly, the velocity dispersion of galaxies is also anisotropic, due to the clusters of galaxies being rotating and having angular momentum. The velocity dispersion for the galaxies in clusters of galaxies is about $\langle V \mid\rangle / c \approx(1-2) \times 10^{-3}$. How much is the anisotropy of the clusters of galaxies? To date, the anisotropy extent of the velocity dispersion for the galaxies in clusters of galaxies has not been well determined because it is very difficult to measure the proper motion of very remote galaxies. It is reasonable to estimate the anisotropy of the galaxies in 
clusters of galaxies, which is estimated as $(1-5) \%$. Thus, $\langle|V|\rangle / c \approx(1-6) \times 10^{-5}$.

The logical relationship of our idea is given as following: The anisotropy of the velocity dispersion for the galaxies in clusters of galaxies is estimated as $(1-6) \times 10^{-5} \mathrm{C}$ as given a small perturbation via the Einstein field equation and a small anisotropic perturbation of metric for the expanding universe (about $\left.(1-6) \times 10^{-5}\right)$. Then, utilizing the Friedmann equation, we get a small anisotropic perturbation of the cosmic scale, $\alpha(t) \approx(1-6) \times 10^{-5}$, and obtain a small anisotropic perturbation of the CMB temperature (i.e., about $\left.(1-6) \times 10^{-5}\right)$ by Equation (6). Finally, we can get the observational data of WMAP and Planck satellites by fitting the above information.

Thus, we may come to a conclusion that the indirect observational evidence of 'Accelerated expansion of the universe' from fitting the WMAP and Planck satellites's observational data is not reliable. It has to be reinvestigated at least.

\section{Observational Evidence for the Anisotropy of the Velocity Space}

For the standard CMB anisotropy, we give the two fundamental assumptions as follows. The first assumption is the initial fluctuations, which are statistically isotropic. The second assumption is the Gaussian, which are rigorously tested by using maps of the CMB anisotropy from the Planck satellite (Planck et al. 2014).

By using a fiducially $\Lambda$-CDM model and incorporating essential aspects of the Planck measurement process, four independent estimates of the $\mathrm{CMB}$, which are compared with simulations have been obtained. The above two assumptions are derived from results of it. From isotropy to be robust against component separation algorithm, mask choice, and frequency dependence, Planck et al. (2014) [18] found the deviations. Although these analyses represent a step forward in building an understanding of the anomalies, a satisfactory explanation based on physically motivated models is still lacking. By using observations from the Planck satellite, Planck et al. (2014) test the statistical isotropy and Gaussianity of the CMB anisotropies.

Based on the full Planck mission for temperature, which includes some polarization measurements, they obtained some results. For the temperature anisotropies, they found excellent agreement between results based on these sky maps over both a very large fraction of the sky and a broad range of angular scales. However, the theoretic fitting the WMAP (and Planck) satellite observational data is not reliable due to the following reason that an important physical factor has not been taken account up to date, in which the velocity space is not isotropic.

Based on the full Planck temperature mission, which included some polarization measurements, they obtained some results. For the temperature anisotropy, between results based on these sky maps over a very large fraction of the sky and a broad range of angular scales, an excellent agreement has been found by them. However, the theoretically fitting the WMAP (and Planck) satellite observational 
data is not reliable, because up to date they have not taken into account an important physical factor, which is that the velocity space is not isotropic.

Except a large pool spot near the north pole of the Galaxy, the observations from WMAP (2008) and Planck (2013) show another important observational evidence for the anisotropy of the velocity space. This is a question on the axis of evil. We cite the results of Planck et al., (2014) in Table 1 [18]. The directions (i.e., galactic longitude (l) and galactic latitude (b)) of orientation vectors for quadrupole and octopole are given in columns 2 and 3 in Table 1 . The angular distances of orientation vectors for quadrupole and the octopole are given in column 4. We can extracted the low multipole orientations from different separated $\mathrm{CMB}$ maps, obtained from maximizing the angular momentum dispersion. Between the orientation vectors of the quadrupole and octopole, the absolute values of scalar-product are given in the second last column. One can see that the latter is uniformly distributed on the interval $[0,1]$ in an isotropic universe. The probability of such an alignment (or stronger than that) to occur will be given in the last column when the universe is really isotropic.

From Table 1, one can see that our universe is not isotropic. However, Planck et al., $(2014,2016)$ [18] [19] had not understood its physical reasons in their papers. Our analysis above on the anisotropic of the velocity dispersion of galaxies from the velocity space of galaxy clusters shows that the velocity space is also anisotropic. These ideas may be one of causes for this phenomenon.

\section{Summary and Discussions}

In summary, we conclude that the anisotropy from the velocity space will inevitably lead to the anisotropy of CMB temperature distribution. So far, people have not yet studied the effect of this factor in detail. However, some scholars arbitrarily conclude that this is just the indirect evidence of the cosmic acceleration due to the fact that it is well fitted to the accelerating expansion model of the universe. We think that this is not serious and proper.

Besides, it is well known that the mass-density distribution structure of remote galaxies is a network-like shape. This shows that it is not completely isotropic in the space. The anisotropy of the mass-density distribution structure of remote galaxies is more than $10^{-5}$. Similar to the analysis above for the perturbation in the velocity space, we may take the small anisotropy (a small difference) of the mass density distribution in the space. A perturbation of the scaling factor

Table 1. Planck 2013 results. XXIII. Isotropy and statistics of the CMB (e.g., [18] [19]).

\begin{tabular}{cccccc}
\hline method & quadrupole (1, b) & octopole $(1, \mathbf{b})$ & ang.distance & scalarproduct & probability \\
\hline C-R & $(228.2,60.3)$ & $(246.1,66.0)$ & 9.80 & 0.985 & 0.019 \\
NILC & $(241.3,77.3)$ & $(241.7,64.2)$ & 13.1 & 0.974 & 0.033 \\
SEVEM & $(242.4,73.8)$ & $(245.6,64.8)$ & 9.08 & 0.988 & 0.016 \\
SMICA & $(238.5,76.6)$ & $(239.0,64.3)$ & 12.3 & 0.977 & 0.032 \\
\hline
\end{tabular}


will be caused by the perturbation of the mass density distribution in the space through the energy momentum tensor in the Einstein field equation, while a perturbation of the $\mathrm{CMB}$ temperature will be also caused by the perturbation of the scaling factor through the relation of $\alpha(t) T(t)=$ const.

On baryon acoustic oscillation (BAO) theory, in the popular idea, another indirect evidence for the accelerating expansion of the universe comes from some baryon acoustic oscillation (BAO) theories. However, the baryon acoustic oscillation (BAO) theories are actually the cosmic density wave theories and they are really the linear perturbation theories of cosmic dynamics, including the Einstein field equation. A relation of the cosmic peak density and matter with red-shift may be obtained by the dispersion relation in the linear perturbation theory.

On the other hand, the effect of the non-linear perturbation theory cannot be neglected in the very early universe, because the Einstein's gravity is much stronger than the Newtonian gravity. So the linear perturbation theory of cosmic dynamics cannot get the conclusion of the accelerated expansion of the our universe. This is just of our idea.

\section{Acknowledgements}

This work was supported in part by the NSFC under grants 11965010, 11565020, and the Natural Science Foundation of Hainan Province under grants 2019RC239, 118MS071, 114012 and the Counterpart Foundation of Sanya under grant 2016 PT43, 2019PT76, the Special Foundation of Science and Technology Cooperation for Advanced Academy and Regional of Sanya under grant 2016YD28, the Scientific Research Starting Foundation for 515 Talented Project of Hainan Tropical Ocean University under grant RHDRC201701.

\section{Conflicts of Interest}

The authors declare no conflicts of interest regarding the publication of this paper.

\section{References}

[1] Luca, A. and Shinji, T. (2010) Dark Energy: Theory and Observation. Cambridge University Press, London.

https://ui.adsabs.harvard.edu/abs/2010deto.book.....A/abstract

[2] Riess, A.D., Filippenko, A.V., Challis, P., et al. (1998) Observational Evidence from Supernovae for an Accelerating Universe and a Cosmological Constant. The Astronomical Journal, 116, 1009-1038. https://iopscience.iop.org/article/10.1086/300499 https://doi.org/10.1086/300499

[3] Schmidt, B.P., Suntzeff, N.B., Phillips, M.M., et al. (1998) The High-Z Supernova Search: Measuring Cosmic Deceleration and Global Curvature of the Universe Using Type Ia Supernovae. The Astrophysical Journal, 507, 46-63. https://iopscience.iop.org/article/10.1086/306308 https://doi.org/10.1086/306308

[4] Perlmutter, S., Aldering, G., Goldhaber, G., et al. (1999) Measurements of $\Omega$ and $\Lambda$ 
from 42 High-Redshift Supernovae. The Astrophysical Journal, 517, 565-586.

https://iopscience.iop.org/article/10.1086/307221

https://doi.org/10.1086/307221

[5] Guy, J., Astier, P., Baumont, S., et al. (2007) SALT2: Using Distant Supernovae to Improve the Use of Type Ia Supernovae as Distance Indicators. $A$ and $A, 466$, 11-21. https://www.aanda.org/10.1051/0004-6361:20066930 https://doi.org/10.1051/0004-6361:20066930

[6] Amanullah, R., Lidman, C., Rubin, D., et al. (2010) Spectra and Hubble Space Telescope Light Curves of Six Type Ia Supernovae at $0.511<\mathrm{z}<1.12$ and the Union2 Compilation. The Astrophysical Journal, 716, 712.

https://iopscience.iop.org/article/10.1088/0004-637X/716/1/712/pdf https://doi.org/10.1088/0004-637X/716/1/712

[7] Phillips, M.M. (1993) The Absolute Magnitudes of Type IA Supernovae. Astrophysical Journal Letters, 413, L105.

https://ui.adsabs.harvard.edu/abs/1993ApJ...413L.105P/abstract https://doi.org/10.1086/186970

[8] Kang, Y.J., Kim, Y.L., Lim, D., et al. (2016) Early-Type Host Galaxies of Type Ia Supernovae. I. Evidence for Downsizing. The Astrophysical Journal Supplement Series, 223, 7. https://iopscience.iop.org/article/10.3847/0067-0049/223/1/7 https://doi.org/10.3847/0067-0049/223/1/7

[9] Kang, Y.J., Lee, Y.W., Kim, Y.L., et al. (2020) Early-Type Host Galaxies of Type Ia Supernovae. II. Evidence for Luminosity Evolution in Supernova Cosmology. The Astrophysical Journal, 889, 8.

https://iopscience.iop.org/article/10.3847/1538-4357/ab5afc https://doi.org/10.3847/1538-4357/ab5afc

[10] Isern, J. (2010) Type Ia Supernova: Observations and Theory. Proceedings of the 11 th Symposium on Nuclei in the Cosmos, Heidelberg, 19-23 July 2010, 66. http://pos.sissa.it/cgi-bin/reader/conf.cgi? confid $=100$

[11] Ropke, F.K., Hillebrandt, W., Kasen, D., et al. (2010) Modeling the Diversity of Type Ia Supernova Explosions. Numerical Modeling of Space Plasma Flows: ASTRONUM-2009 ASP Conference Series, Vol. 429, 142-147. http://articles.adsabs.harvard.edu/pdf/2010ASPC..429..142R

[12] Kasen, D. (2010) The Supernova Has Two Faces. Nature, 466, 37-38. https://ui.adsabs.harvard.edu/link gateway/2010Natur.466...37K/doi:10.1038/46603 $\underline{7 \mathrm{a}}$ https://doi.org/10.1038/466037a

[13] Maeda, K., Benetti, S., Stritzinger, M., et al. (2010) An Asymmetric Explosion as the Origin of Spectral Evolution Diversity in Type Ia Supernovae. Nature, 466, 82-85. https://doi.org/10.1038/nature09122

[14] Wang, X.F., Wang, L.F., Alexei, V.F., et al. (2013) Evidence for Two Distinct Populations of Type Ia Supernovae. Science, 340, 170-173.

https://science.sciencemag.org/content/340/6129/170 https://doi.org/10.1126/science.1231502

[15] Nielsen, J.T., Guffanti, A. and Sarkar, S. (2016) Marginal Evidence for Cosmic Acceleration from Type Ia Supernovae. Scientific Reports, 6, Article No. 35596. https://ui.adsabs.harvard.edu/link gateway/2016NatSR...635596N/doi:10.1038/srep $\underline{35596}$ https://doi.org/10.1038/srep35596

[16] Weinberg, S. (2008) Cosmology. Oxford University Press, Oxford. 
https://ui.adsabs.harvard.edu/abs/2008cosm.book.....W/abstract

[17] Weinberg, S. (1972) Gravitation and Cosmology: Principles and Applications of the General Theory of Relativity. Wiley-VCH, Hoboken, 688.

https://ui.adsabs.harvard.edu/abs/1972gcpa.book.....W/abstract

[18] Alves, J., Bertout, C., Combes, F., et al. (2014) Planck 2013 Results. Astronomy and Astrophysics, 571, E1.

https://www.aanda.org/articles/aa/pdf/2014/11/aa25195-14.pdf

[19] Alves, J., Combes, F., Ferrara, A., et al. (2016) Planck 2015 Results. Astronomy and Astrophysics, 594, E1.

https://www.aanda.org/articles/aa/pdf/2016/10/aa29543-16.pdf 